\title{
Development of Non-Fourier Thermal Attitude for Three-Dimensional and Graphene-Based MOS Devices
}

\author{
Zahra Shomalia,*, Abbas Abbassi ${ }^{\mathrm{b}}$, Jafar Ghazanfarian ${ }^{\mathrm{c}}$ \\ ${ }^{a}$ Mechanical Engineering Department, Amirkabir University of Technology, 424 Hafez \\ Avenue, P.O. Box 15875-4413, Tehran, Iran. \\ ${ }^{b}$ Mechanical Engineering Department, Amirkabir University of Technology, 424 Hafez \\ Avenue, P.O. Box 15875-4413, Tehran, Iran. \\ ${ }^{c}$ Mechanical Engineering Department, Faculty of Engineering, University of Zanjan, \\ Zanjan, Iran.
}

\section{Abstract}

In this paper, the intact subject of 3-D MOSFETs is studied. Three wellknown 3-D MOSFET nano-devices, including a common three dimensional silicon MOSFET, the FinFET, and the Tri-Gate, are dealt. Firstly, by implying the three dimensional non-linear Dual-Phase-Lag method, the obtained results are verified with the existent data. Contemporaneously, the important parameter $B=\tau_{t} / \tau_{q}$, which is prerequisite for DPL modeling, is found for the 3-D silicon. The adjusted parameter $B$, is used to study the 3-D transistors with temperature-dependent thermal characteristics. It is found that taking into account such relevancy, makes the overall trends of distributions unmodified. Finally, the effect of contemplating the graphene sheet heat remover in a 3-D Tri-Gate MOSFET is investigated. Notably, the graphene makes the process of cooling the transistor get accelerated. It is obtained that $40 \mathrm{ps}$

*Corresponding author. Tel.: +98 (24)33054142

Email addresses: shomali.zahra@gmail.com (Zahra Shomali), abbassi@aut.ac.ir (Abbas Abbassi), j.ghazanfarian@znu.ac.ir (Jafar Ghazanfarian)

Preprint submitted to applied thermal engineering

May 13, 2016

(C) 2016. This manuscript version is made available under the Elsevier user license http://www.elsevier.com/open-access/userlicense/1.0/ 
after switching off the heat generation region, the value of the peak temperature of a 3-D Tri-Gate transistor along the center-line with a graphene heat remover, becomes $20 \%$ lower than that of a common 3-D Tri-Gate MOSFET. This finding shows that the Fourier calculations, overestimate the results. Keywords: , Three Dimensional, Non-Fourier, Nanoscale Heat Transfer, MOSFET, FinFET, Tri-Gate

\section{Nomenclature}

$B \quad$ phase-lag ratio

$k \quad$ thermal conductivity $\left(W K^{-1} m^{-1}\right)$

$Q \quad$ Volumetric heat source $\left(W m^{-2}\right)$

$x \quad$ direction $(m)$

$z \quad$ direction $(m)$

$k_{B T} \quad$ Boltzmann constant $\left(\mathrm{m}^{2} \mathrm{kgs}^{-2} \mathrm{~K}^{-1}\right)$

$\gamma \quad$ dummy variable $(\mathrm{x}, \mathrm{y}, \mathrm{z})$

$t \quad$ time $(s)$

Kn Knudsen number

\section{Greek}

$\alpha \quad$ BC coefficient $\left(\mathrm{kgm}^{-3}\right)$

$\lambda \quad$ mean free path of phonons $(m)$

$\tau \quad$ relaxation time $(s)$

\section{superscript}

* non-dimensional condition

\section{subscript}

q heat flux

t gradient temperature
$C \quad$ Volumetric heat capacity $\left(\mathrm{Jm}^{-3} \mathrm{~K}^{-1}\right)$

$v \quad$ average velocity of sound $\left(m s^{-1}\right)$

$q \quad$ heat flux $\left(W m^{-2}\right)$

$y \quad$ direction $(m)$

$E_{s s} \quad$ Steady-State error

$n \quad$ density and direction normal to boundary

$Y \quad$ dummy variable $(\mathrm{q}, \mathrm{T})$

$T$ Temperature $(K)$

$\omega \quad$ under-relaxation factor

r coordinate

i counter 


\section{Introduction}

The silicon metal-oxide-semiconductor field effect transistor (MOSFET), has been the main block of the semiconductor industry since 1960 [1]. The size of these MOS transistors has been decreased continuously over the past decades to a mean size of few-ten nanometer in the most recently designed MOSFETs. Hence, as the transistor dimensions have reached the nanoscale regime, the classical equations of continuum mechanics are ineffective. Consequently, the traditional continuum heat diffusion equation is not a good choice for nanoscale heat transfer investigation. Also, the usage of the atomistic methods for complex geometries due to their time-consuming, difficulties, and complex characteristic is not faultless [2]. Besides, the thermal issues such as self-heating in MOSFETs, are nowadays an important contention in design and optimization considerations of these SOI nano-devices $[3]$.

Accordingly, the innovative models with less computational cost and more simplicity have been introduced to rectify the outcome of classical equations such as Fourier's law [4-9]. In fact, the Fourier's law encounters serious challenges in heat conduction prediction at ultra-small temporal and spatial scales. Several theoretical and experimental studies have reported that nanoscale heat conduction violates Fouriers law [10-12]. Consequently, the thermal properties deviate significantly from that of the normal sizes [13]. It is important to note that the failure of the Fouriers law in nanomaterials with phonon mean free path much shorter than the characteristic length also occurs [14]. This can be due to the ultrahigh-speed heat flux, resulting from the exceedingly high temperature gradient or the very-low cross-section area. In 
such situations, although the characteristic length is within nanoscale range, but the continuum assumption is still valid. This presumption is not fundamentally explainable but according to the potentiality for using in technical and engineering applications is of great interest. Several theories have been developed to describe the heat transport in nanomaterials, including phonon dynamics [15], phonon hydrodynamics [16], thermomass theory [17], and phase lagging models. In the meantime, the dual-phase-lag (DPL) model as a new modified constitutive equation to investigate the non-Fourier heat transport in various cases such as nanoscale MOSFETs [18-20], ultra-fast laser-pulsed processes [21-24], living tissues [25-27], and Carbon nanotube [28-31] has attracted attentions. Due to the simpleness, straightforwardness, flexibility, and accurateness of this method, it can be a good choice for the study of transistors with various geometries.

Additionally, due to the complicated conditions existed in the transistors, simulations of these nano-devices considering real conditions seems to be necessary and worthwhile. Although, there have been many works dealing with the transistors as one- or two-dimensional nano-systems [32-34], there are few works, which have claimed to investigate the more realistic threedimensional transistors.

In the work by Nasri et al. [35], a three-dimensional Tri-Gate is investigated using the single-phase-lag model. This work implies many limitations and approximations. Above the fact that the single-phase-lag model (Cattaneo-Vernote model) does not predict the behavior of nano-scale systems precisely, the coefficients used in the temperature-jump boundary condition are the ones used for a two-dimensional system. These two coefficients 
are obtained, respectively, by the two-dimensional results of the DPL model [34] and SPL model [36] and, consequently, are applied to the single-phaselag simulation of three-dimensional cases. In the present work, the theedimensional non-linear dual-phase-lag model is used to simulate the realistic structure of the several different three-dimensional silicon MOS devices.

As the simulation of 3-D MOSFETs are completed, the effect of inserting the graphene heat spreader between the substrates of the transistor, on the thermal behavior of these nano-structures are investigated. In the last decade, graphene, a 2-D material with odd properties and high electrical and thermal conductivity has attracted lots of attention. First, the graphene sheet was proposed to replace the silicon in the MOSFET industries. Zero bandgap of graphene leads to low on-off current ratios of 2 to 20, which makes the large-area-graphene unsuitable candidate for MOSFET technology. The current ratio can be increased by shrinking the graphene sheet in one-dimension and making the graphene nanoribbons. Although the thermal conductivity of narrow graphene ribbons decreases, there are some proposal for the use of these nano-materials for making new generation of transistors $[37,38]$.

Despite the unsuitability of large-area-graphene sheet for the substitution of silicon in SOI transistors, its extremely high intrinsic thermal conductivity suggests to use this two-dimensional material as an appropriate candidate in thermal management of the nano-electronics devices. In recent years, there have been several works proposing the graphene sheet as a lateral heat spreader. But, even though the thickness of the graphene sheet is 0.324 nanometers and hence the non-Fourier methods should be applied to study 
these nano-systems, to the best of our knowledge, all of researches used the Fourier technics, and ignored the important non-Fourier effects [39-42]. In the present work, by the numerical modeling of the MOSFET including a graphene sheet between the $\mathrm{Si}$ and $\mathrm{SiO}_{2}$ substrates, using the non-linear 3-D-DPL model, the real role of graphene heat spreader is investigated.

In the current study, first, the accuracy of the new 3-D DPL numerical modeling is investigated. By using the fact that enlarging the third dimension, the $3-\mathrm{D}$ results should reach that of the $2-\mathrm{D}$ problem, the findings are verified. Secondly, by comparing the 3-D-DPL simulation of the FinFET and Tri-Gate silicon MOSFETs without a heat spreader with the data obtained from the Lattice-Boltzman simulation of three-dimensional MOSFETs [43], the three-dimensional coefficients of the 3-D DPL model are computed. Then, the realistic modeling of transistors with a graphene sheet heat spreader is performed by using 3-D nonlinear/linear DPL method.

\section{Geometry and boundary conditions}

The goal of this paper is to present the non-Fourier investigation of more realistic Three-Dimensional transistors. Also, the effects of using a graphene sheet as a heat spreader in the SOI MOSFETs such as typical 3-D Tri-Gates are treated. Hence, four different three-dimensional MOSFET devices are

modeled in various circumstances as shown in Figs. 1(a-c). The heat source and boundary conditions are chosen in similarity to a real 3-D MOSFET.

\subsection{Case I: A 3-D common silicon MOSFET}

In case I, a three dimensional silicon transistor of size 100, 1000, and 50 $n m$, respectively, in $x, y$, and $z$ direction with constant initial temperature 
of $300 K$ is investigated. As it is manifested in Fig. 1(a), the heat generation zone of $10 \mathrm{~nm} \times 1000 \mathrm{~nm} \times 10 \mathrm{~nm}$ is created in the middle of the cubic MOSFET due to the operation of the transistor. This functionality results in the creation of the hot spot and the rearrangement of the temperature distribution in the MOSFET. The heat transport is studied while the heat generation zone produces volumetric heat during the whole time of the simulation. All boundaries except the bottom boundary, at which the temperature is kept constant at room temperature, are adiabatic. In the limit of large depth transistor, the results of our 3-D DPL modeling are verified with the data presented in [34]. Hence, this case is proceeded for the 3-D MOSFET with considering constant thermal parameters of the bulk silicon. Further, the size of the heat generation zone, $L_{H}$, which shows the characteristic length of the simulation, is the most important geometrical parameter of heat conduction.

\subsection{Case II: A 3-D FinFET transistor}

The schematic of the FinFET, which has been investigated in [43] is shown in Fig. 1(b). The silicon channel is a $22 \mathrm{~nm} \times 100 \mathrm{~nm} \times 200 \mathrm{~nm}$ region. The heat generation zones are two cubic volumes with $10 \mathrm{~nm}$ width, $100 \mathrm{~nm}$ height, and $200 \mathrm{~nm}$ depth. Also, as it is displayed in Fig. 1(b), the

substrate is a $100 \mathrm{~nm} \times 100 \mathrm{~nm} \times 200 \mathrm{~nm}$ zone where two cubic regions of $\mathrm{SiO}_{2}$ substrate are located in its both sides and the remaining compartment is a silicon substrate. The size of the each cubic $\mathrm{SiO}_{2}$ substrate is $39 \mathrm{~nm} \times$ $32 \mathrm{~nm} \times 200 \mathrm{~nm}$. Moreover, all boundaries are adiabatic except the bottom plane boundary, which is kept at the ambient temperature. 


\subsection{Case III: A 3-D Tri-Gate transistor}

The typical Tri-Gate MOSFET, is shown in Fig. 1(c). The silicon channel length is $22 \mathrm{~nm}$. Further, the heat generation region of $Q=10^{19} \mathrm{~W} / \mathrm{m}^{3}$, is an n-shaped volume with $10 \mathrm{~nm}$ thickness, and $100 \mathrm{~nm}$ depth. The other segments of the transistors such as the substrate shape, are similar to that of FinFETs. In analogy to the previous cases, all the boundaries except the bottom one, which is kept at $300 K$, are adiabatic.

\subsection{Case IV: A Tri-Gate MOSFET with graphene sheet heat remover}

At last, a typical Tri-Gate MOSFET with a graphene heat spreader is investigated. To look more closely, the effects of putting the newly added layers on the mechanism of the heat transfer are studied. In particular, the influence of the graphene sheet insertion, on the maximum value of the hotspot is concerned. The geometry is defined in similarity to the work by

Subrina et al [39], where the graphene layers are situated between the $\mathrm{SiO}_{2}$ and $S i$ substrates. The thickness of the each graphene sheet is $0.345 \mathrm{~nm}$. Similar to the foregoing samples, the heat flux of $Q=10^{19} \mathrm{~W} / \mathrm{m}^{3}$, is applied to the n-shaped region of the silicon channel. All boundaries except the bottom boundary are adiabatic. Hence, the most of the heat generated inside the cubic MOSFET is conducted toward it. In addition, two other sets of circumstances are also explored. First, the case in which the graphene boundaries are also adiabatic, is researched. Then, the transistor is considered to exchange heat from right and left boundaries of the graphene sheet. In other words, two left and right sides of the graphene is kept at the ambient temperature. 


\section{Mathematical modeling}

In this paper, the three-dimensional non-linear DPL modeling is used for the investigation of heat conduction in 3-D MOSFETs with and without graphene sheet heat spreader. The way of using the phase-lagging behavior for heat transfer investigation, firstly, has been opened by the proposition of the single phase-lagging model (CV model), $\vec{q}\left(t+\tau_{q}, \vec{r}\right)=-k \nabla T(t, \vec{r})$, by Cattaneo [44] and Vernotte [45]. In this model, the heat flux vector, $\vec{q}$, is the effect of temperature gradient. Also, $\tau_{q}$, the relaxation time, is the time delay between these happenings and $k$ is the thermal conductivity. In 1995, Tzou announced a non-Fourier phenomenological equation for heat conduction in which, the heat flux vector somewhere in the material at the time of $\tau_{q}$, is related to the temperature gradient at the same point at time $\tau_{t}$ via the formula $[46,47]$,

$$
\vec{q}\left(t+\tau_{q}, \vec{r}\right)=-k \vec{\nabla} T\left(t+\tau_{t}, \vec{r}\right)
$$

where, $\tau_{q}$ and $\tau_{t}$ are standing for the heat flux and temperature gradient phase lags, respectively. Both parameters are positive and fundamental properties of the material, and may be determined experimentally or numerically. Taylor expanding Eq.1 in three-dimension with respect to time yields:

$$
q_{\gamma}+\tau_{q}(T) \frac{\partial q_{\gamma}}{\partial t}=-k(T)\left[\frac{\partial T}{\partial \gamma}+\tau_{t}(T) \frac{\partial}{\partial t} \frac{\partial T}{\partial \gamma}\right]
$$

where, $\gamma$ is the representative of the $x, y$ and $z$ component of coordinates.

Furthermore, considering $C(t)$ as the volumetric heat capacity of the material, the energy equation is written as,

$$
\frac{\partial q_{x}}{\partial x}+\frac{\partial q_{y}}{\partial y}+\frac{\partial q_{z}}{\partial z}=-C(T) \frac{\partial T}{\partial t}+Q
$$


In the present study, three Taylor-expanded DPL equation (Eq. 2), which is coupled with the energy equation, is used simultaneously to investigate the heat transport process $[48,49]$. For some cases of transistors, the temperature-dependent thermal properties are considered.

Looking more precisely to the DPL and energy equations by taking the derivative of Eq. 2 with respect to the one of the components like $x$, it can be seen that the result of taking into account the temperature-dependent properties causes the appearance of the terms such as $\frac{\partial \tau_{q}}{\partial x}$. This corresponds to the non-monotonous distribution of $\tau_{q}$. It is conspicuous that the components of the heat flux vector can not be removed between the three DPL equations and the energy equation, and consequently, these equations should be separately solved. In addition, the boundary-phonon scattering is considered by exerting the following mixed-type boundary condition in analogy to the work done by Ghazanfarian and Abbassi [33, 34],

$$
T_{s}-T_{w}=-\left.\alpha K n \frac{\partial T}{\partial n}\right|_{\Omega}
$$

Here, $T_{s}$ and $T_{w}$ are respectively, the temperature-jump of the wall and the boundary temperature. $\alpha$ is a non-dimensional modifiable coefficient, $\Omega$ includes all boundaries of the solution domain, and $n$ is the direction of the outward normal vector of the boundary. Also the Knudsen number, $K n$, is the ratio of the mean free path to the size of the system. Hence, the above equation is the boundary condition applied to the all open bottom boundaries. Further all the other adiabatic boundaries fulfill the following condition:

$$
\vec{q} \cdot \vec{n}=0 .
$$


More importantly, the non-dimensional parameter $B$, which is the ratio of the temperature relaxation time, $\tau_{t}$, to the heat flux relaxation time, $\tau_{q}$, is defined. Then, $B \tau_{q}$ is substituted for $\tau_{t}$ in Eq. 2 .

The values of two unknown parameters $\alpha$ and $B$, for each instant of time, have been computed and presented for 1-D and 2-D silicon devices [33, 34]. In this work, for some three-dimensional silicon structures, the values of the two unknown parameters $\alpha$ and $B$, for each instants of time, is determined in a such way that the results of the 3-D DPL model is in agreement with the solutions of the lattice Boltzmann equation. It is obtained that the value of the parameter $B$, changes with time and is given by the following relations

$$
B=0.25 \Theta\left(20-t^{*}\right)+1.9 \Theta\left(t^{*}-20\right)
$$

where, $t^{*}$ is the dimensionless time, which is scaled to $1 \mathrm{ps}$. As it is shown, a simple Heaviside step function is found for parameter $B$. Also the unified $B=$ 0.25 , for all times is also checked. It is attained that this unique $B$ roughly predicts the overall behavior of the temperature and heat flux distributions. This non-zero $B$, manifests that the thermal behavior deviates from the Fourier's law. Inevitably, replacing the Fourier's law by the non-Fourier methods such as the present 3-D DPL model is an essential development. Just as the time passes, $B$ increases to prevent the reduction of the effect of the phase-lagging term. On the other hand, as the system reaches the steady state, the effect of the phase-lagging term diminishes. As a result, the value of the parameter $B$ has a limitation. 


\section{Numerical considerations}

In the following subsections, the numerical issues in modeling the different four cases of MOSFETs are discussed thoroughly.

\subsection{Applied method}

In the present work, the 3-D DPL and energy equations are discretized simultaneously by using the fully implicit finite difference scheme with the central discretization of all derivatives associated with the Dirichlet, Neumann, and Robin Boundary conditions. Solving three components of DPL model along with the energy equation makes sense since four first-order coupled partial differential equations (PDE)s are solved instead of one single third-order PDE. This simplification results in some numerical difficulties, which are thoroughly explained in Shomali and Abbassi [48].

\subsection{Convergence contemplation}

Owing to the utilization of the mentioned method for modeling the desired structures, and also the appearance of the non-linearity due to the consideration of the temperature-dependent thermal properties, the underrelaxation factors are introduced as $\omega_{T}$ and $\omega_{q}$. These factors are used in the beginning of a new iteration for $Y=T, q$ via the following relations, $Y_{i}=\omega_{Y} Y_{i}+\left(1-\omega_{Y}\right) Y_{i-1}$. It is obtained that the value of these factors strongly relies on the value of $B$. As $B$ becomes larger, the relaxation factors, which make the convergence of the solution easier get smaller. Strictly speaking, it is found that, when $B$ is smaller than 0.1 , the relaxation factors $\omega_{q, T} \leq 0.8$ are sufficient to satisfy the heat flux and temperature convergence. Further-

more, taking $\omega_{q, T} \leq 0.1$ is adequate for fulfilling the convergence condition 
of the problem with $0.1<B<0.45$. For greater $\mathrm{B}$, the under relaxation factors are needed to decrease to 0.05 or even smaller. Obviously, the optimum computational time is reached by the consideration of maximum values of the indicated $\omega_{T, q}$. It is important to note that by taking into account the temperature-dependent thermal properties, smaller under-relaxation factors should be used.

\subsection{Grid independency check}

Moreover, the tolerance of residuals of temperature and heat flux on convergence relative to the infinity norm is set to $10^{-10}$. Further, the meshindependence test is checked for all cases. As it is seen in table. 1, the mesh for a common 3-D MOSFET is a uniform three-dimensional $101 \times 101 \times 201$ numerical mesh, which is sufficient for generating the mesh-size-independent results for all times. For the FinFET and Tri-Gate structures, the $x, y$, and $z$ dimensions of the channel are discretized into 22, 201, and 101 nodes. Also, the substrate is meshed by the grid of $101 \times 201 \times 101$ nodes. Besides, the time-step is taken to be $10^{-2} \mathrm{ps}$. It is attained that these discretizations are the appropriate ones for meshing the structures such that they make the results, mesh- and time-step size independent.

\section{Results and discussions}

The present 3-D DPL model is employed to study the temperature and heat flux distributions for all four types of 3-D MOSFETs. Also, each of these 3-D transistors are dealt for various conditions. In the forthcoming sections, the obtained results are discussed. 


\subsection{Case $I$}

The case I explores the modeling of a common 3-D MOSFET by imposing three-dimensional DPL method. The proposed transistor is the same as that of considered in [34], which is a cube with the cross section of $100 \mathrm{~nm} \times 50 \mathrm{~nm}$, and the depth of $1 \mu \mathrm{m}$. The thermal parameters of the bulk silicon at $300 \mathrm{~K}$ are given in table 2. As it is shown in Figs. 2(a,b), the results obtained from the new modeling of the 3-D DPL method fit well with the results displayed in [34]. This shows the proficiency and accuracy of the present $3-\mathrm{D}$ numerical simulation. To be more precise it is found that for $t=10 \mathrm{ps}$, when the out-of-plane dimension is larger than $22 \mathrm{~nm}$, the heat flux and temperature distribution plots along the $y$ coordinate are similar to that of the 2-D MOSFET. In other words, the third dimension will not affect much on results when it is greater than $22 \mathrm{~nm}$. For the $y$ values less than $22 \mathrm{~nm}$, the effects related to the three-dimensionality appear such that the behavior of the heat and temperature profiles are different for all values of the $y$ coordinate.

\subsection{Case II}

In this section, the FinFET transistor discussed in [43], is studied. By the verification of results obtained from the 3-D DPL non-linear method, with the atomistic results presented in [43], the appropriate value of key parameter $B$, which is the ratio of $\tau_{t}$ to $\tau_{q}$ can be tuned. In the mentioned work, the results are acquired by implying the finite-volume-lattice-Boltzmann method. In equivalence to the nano-system remarked in [43], the heat generation zone of the FinFET transistor is on for the first 10 ps switching time, then the transistor starts to get cool. As Fig. 3 suggests, in similar conditions, by 
searching for the correct value of $B$ for Silicon and Silicon-dioxide, the temperature and heat flux plots match almost well with those of Samian et al.

In agreement with the previous studies, the DPL model predicts the heat transfer almost better for lower instants of time $[48,49]$. It is important to note that the non-Fourier effects appear for the ultra-fast and micro/nano sized processes. Hence, as the time passes and gets larger relative to the characteristic time, the behavior of the system starts to deviate from the non-Fourier etiquette and it becomes nearer to the Fourier behavior. For this reason, parameter $B$, which is the principal factor to fit the results of the DPL to the lattice-Boltzmann, enhances as time increase.

Also, as time evolves, a discrepancy between our results and those of Samian et al. appears. As it is manifested in Fig. 4, a breaking which is presented in the heat flux plots of $t \geq 15 \mathrm{ps}$, come into sight for greater time in our results. The Fig. 5 displays the heat flux profile at $t=20 \mathrm{ps}$. It is obtained that for times greater than $15 \mathrm{ps}$, the heat flux value drops more quickly due to the enhancement of phase-lags ratio. Moreover, the heat flux is plotted for the unified parameter $B=0.25$. As it is seen, this unified $B$ does not predict the results accurately. Hence, the time-dependent parameter $B$ is more preferable in the numerical investigation of 3-D Silicon MOSFETs.

In the next step, the effect of considering the more realistic temperaturedependent thermal properties is investigated. The temperature-dependent thermal characteristics of the bulk silicon are presented in [49]. Two different cases of a 3-D FinFET are studied. The first one is the three-dimensional FinFET device with an all-time heat generation zone, while the second con- 
figuration is the one in which the heat is generated for the first $10 \mathrm{ps}$, and then the cooling process take places. For the first case, as it is shown in Figs. 6(a,b), taking into account such relevance, only changes the value of the obtained temperature and heat flux while it does not drastically modify the trends. This finding is in consistence to the results obtained for the largesized 1-D transistors, and is in contrast to the results obtained for low-sized 1-D transistor [49]. This deduction is somehow guessable due to the fact that while dealing with systems, as the size of the 1-D transistors increases, the influence of such dependency decreases. Further, Figs. 6(c,d) show that the response of the second case to the temperature-relevance thermal parameters is similar to that of the first one. This is also assigned to the attitude of the large-sized nano-structures.

In addition, by enlarging the time, the difference between the temperature profiles obtained by taking into account the temperature-dependent thermal properties, and the one calculated by considering the constant thermal parameters becomes more accentuated. This is because of the way that the thermal conductivity and the specific heat vary with time. Going through time, the temperature gets higher, which results in much larger specific heat and lower thermal conductivity. Hence, as the time increases the temperature, which inversely depends on $C_{p}$, and directly on $k$ decreases much more relative to the case with constant parameters.

\subsection{Case III}

At the next step, the 3-D DPL heat conduction model is used for the investigation of heat transfer in 3-D Tri-Gate transistors. In similarity to the mentioned FinFET, the n-shape region of the present 3-D MOSFET 
is heated for the first $10 \mathrm{ps}$. One of the most important findings is the appearance of a small peak in addition to the main large peak manifested in the heat flux plots of the FinFET MOSFETs. In Figs. 7(b,d), the first small and second large peaks are appeared. These peaks occur at the places where the heat generation zone meets the non-heating region. Since the heating zone is n-shaped, when the heat flux is plotted versus $z$, it is expected that two peaks in the heat flux plots take place at the boundaries between the heat generation region and non-heated zone. Hence, when whole top silicon cube act as heat generation region, the small peak disappears. Also, the temperature distribution manifests a non-monotonous behavior. As it is exhibited in Figs. 7(a,c), the temperature presents an unusual enhancement at the region in which the heat generating existed relative to the other places over the center-line of the transistor. In analogy to the appearance of the small peak in heat flux plots, this growth is also due to the act of the n-shaped heat generation zone.

Just as the time passes, the system starts to move to the condition similar to the FinFET structures. This is due to the switching off the heating zone, which results in equivalence of the Tri-Gate and FinFET transistors in larger times. Consequently, as it is obvious from Figs. 8(b) and 9, after $t=$ 15 ps the second peak in heat flux commences to fade, such that it evanesces completely at 20 ps. Accordingly, this disappearance also takes place for the peak appearing in the beginning of the temperature plot, such that as it is presented in Fig. 8(a), at $t=15 \mathrm{ps}$, this augmentation is completely resolved.

These results confirm that the presence of the third gate in Tri-Gate 
MOSFETs does not make the transistor thermally inefficient. In other words the third gate, which is existed at top of the channel, only mildly changes the heat flux and temperature distributions near the top gate for the first 15 ps. In consequence, one more gate of the Tri-Gate coupled to the two gates of the FinFET transistor, makes the Tri-Gate MOSFET electrically more effective while it leaves the thermal affects such as the value of the hotspot nearly untouched. As a result, it is obtained that the use of TriGate MOSFETs instead of the FinFETs has been more beneficial to the nano-electronic industry.

Besides, the more realistic case of a Tri-Gate with temperature-dependent thermal parameters are investigated. In similarity to the Tri-Gate with constant thermal properties, the heat generation zone is considered to be switched on for the first 10 ps. As it is conspicuous in Figs. 10(a-d), taking into account such relevancy increases the flux of heat flowing toward the bottom boundary of the Tri-Gate, which accordingly decreases the value of the temperature along the center-line. Hence, all the studies considering the MOSFETs with constant thermal characteristics, have over-estimated the value of the temperature in the whole transistor, and in hotspots. Also, it is found by contemplating temperature-dependent thermal properties that the effect of the presence of the third gate on thermal responses of the Tri-Gate, reduces more quickly. To be more precise, as Figs. 10(b,d) illustrate the second peak of the heat flux profile of the transistor with temperature-dependent thermal properties diminishes more promptly such that it completely disappears at $t=15 \mathrm{ps}$. At last, it is obtained that the Tri-Gate transistor with real circumstances is the admirable one for using in the new generation of 
electronic chips.

\subsection{Case IV}

In this part, the other main goal of this paper is implemented. A graphene sheet is added between the silicon and silicon dioxide substrate layers. It is evident that the usage of graphene heat remover effectively decreases the temperature enhancement caused by the generated heat inside the transistor. In the following, the procedure is explained in details. The switching time of the transistor is taken to be $10 \mathrm{ps}$. During this time the $1 \times 10^{19} \mathrm{~W} / \mathrm{m}^{3}$ heat is produced inside the n-shaped region. After $t=10 \mathrm{ps}$, this generated heat starts to spread over the transistor. The case IV deals with the 3-D Tri-Gate MOSFET in the presence of the graphene heat removal. To be more precise, the effect of using few-layer graphene (FLG), say, one to twenty layers of the graphene is investigated. In analogy to the previous case, it has been shown that the thickness of the graphene heat spreader directly affects the heat flux removal from the hotspot zone.

It is obtained that for the first 20 ps of the transient regime, this insertion, nearly does not change the behavior of the temperature and heat flux plots. After $t=20 \mathrm{ps}$, the graphene heat remover slowly exerts influences on the heat flux and temperature distribution of the regions around and at the place of the spreader. As the time passes, the zone which is under the effect of the presence of the graphene gradually enlarges, while the strength of the effect also steadily increases. This finding is easy to understand, since it takes time for the heat generated at the top of the channel to reach to the underlying heat spreader and get perturbed.

This slow influence penetration of the graphene heat spreader is shown 
in Fig. 11(a). It should be emphasized that when the number of graphene layers is less then ten, the use of the heat spreader is not worthwhile. This is due to the fact that adding the graphene sheets to the transistor makes the designing process more complex and expensive while receiving a very little impact on the reduction of the peak temperature rise. On the other hand, the thermal characteristics of the FLG, in the limit of large number of layers tends to that of graphite. As a consequence, due to the increment of the thickness of the transistor, which is in contrast to the spirit of nanoelectronic industry, and also the lose of superior thermal conductivity of the graphene, which is the reason of using this material as a good candidate for heat spreading, the number of graphene layer between the $\mathrm{SiO}_{2}$ and $\mathrm{Si}$ substrate cannot be enlarged non-systematically.

In this paper, it is shown that 10 to 30 graphene sheets are the optimum amount of layers needed as a heat remover. It is attained that this number of layers is not too few such that it could not affect the heat management of the transistors, but also not too many that the problems related to the large number of layers to be appeared.

As it is manifested in Figs. 11(a) and (b), when $t=10 \mathrm{ps}$, the temperature and heat flux profiles of the Tri-Gate MOSFET with and without a heat spreader are almost the same. But as the time passes, the difference between the temperature and heat flux distributions of these two different 3-D MOSFET becomes more apparent. For instance after $100 \mathrm{ps}$, the insertion of the graphene heat remover decreases the peak temperature rise up to nearly $20 \%$. Further, interestingly, at $t=150 \mathrm{ps}$, the graphene-based Tri-Gate presents $50 \%$ reduction in the maximum temperature value relative 
to that of the typical Tri-Gate. Additionally, as it is obvious in Fig. 11(b), the heat flux distribution also varies in the benefit of the reduction of the overall temperature value.

At last, the peak temperature reduction of the other mentioned case of a Tri-Gate MOSFET with a heat spreader is investigated. In similarity to the work by Subrina et al. [39], this 3-D Tri-Gate transistor is considered to exchange heat from right and left boundaries of the graphene sheets. It is acquired that the effect of such graphene sheet open boundary does not show up any distinction. That is to say the temperature profiles of the two various 3-D Tri-Gate transistors including a heat removal segment, are the same.

\section{Conclusions}

The heat transfer issue in three dimensional silicon MOSFET was considered. Three cases of the common 3-D silicon MOSFET, the FinFET transistor, and the Tri-Gate silicon transistor are investigated. The modeling is done by employing the 3-D non-linear DPL method. First, the common 3-D MOSFET and the FinFET transistors are used to verify the results with the existent data. During this verification the parameter $B=\tau_{t} / \tau_{q}$ is adjusted for a 3-D silicon material.

Then the typical Tri-Gate transistor is studied and the results are compared to those of FinFET transistors. The appearance of the relative peak of heat flux in addition to the absolute peak existed in the FinFET, is the main contribution in thermal behavior occurring in the Tri-Gate MOSFET. Also, the effect of considering the temperature dependence of the thermal param- 
eters on the temperature and heat flux distributions of the 3-D transistors are investigated. It is seen that taking into account such relevancy, does not vary the overall behavior of the profiles and it only changes the values of the temperature and heat flux.

At last, the graphene sheet heat remover is added to the 3-D silicon TriGate MOSFET and the non-Fourier effect of placing the graphene sheet is investigated. It is seen that putting the graphene heat spreader between the silicon and silicon dioxide substrates decreases the overall and in particular the maximum peak temperature rise in the hotspot zone up to 50\%. Also, the use of graphene makes the process of device cooling faster. For instance, 100 ps after the heat generation zone gets off, the maximum temperature of a 3-D Tri-Gate transistor with a graphene sheet heat spreader becomes $20 \%$ lower than that of a common 3-D Tri-Gate MOSFET. It should be also mentioned that the results of the Fourier-based studies over-estimates the effect of setting a graphene heat remover, hence, the non-Fourier investigation of such system is necessary.

In conclusion, due to the absence of the study which precisely concerns the 3-D MOSFETs, the corresponding 3-D dual-phase-lag model for the investigation of non-linear nanoscale heat transfer phenomenon in various 3-D MOS devices, such as FinFET and Tri-Gate transistors is evaluated in this paper. Furthermore, as there is no work considering the non-Fourier effects of the setting graphene as a heat spreader in MOSFETs, these non-Fourier consequences are also studied. 


\section{Acknowledgement}

This research is supported by the Iran National Science Foundation (INSF).

\section{References}

[1] E. Pop, K.E. Goodson, Thermal phenomena in nanoscale transistors, J. Heat Transfer 128 (2006) 102.

[2] R.S. Samian, A. Abbassi, J. Ghazanfarian, Transient conduction simulation of a nano-scale hotspot using finite volume lattice boltzmann method, Int. J. Mod Phys C 25 (2014) 1350103.

[3] M.C. Cheng, F. Yu, L. Jun, M. Shen, G. Ahmadi, Steady-state and dynamic thermal models for heat flow analysis of silicon-on-insulator MOSFETs, Microelectron. Reliab. 44 (2004) 381.

[4] W. Dai, R. Nassar, A finite difference scheme for solving the heat transport equation at the microscale, Numer Meth Part D E 15 (1999) 697.

[5] W. Dai, R. Nassar, A compact finite difference scheme for solving a three-dimensional heat transport equation in a thin film, Numer Meth Part D E 16 (2000) 441.

[6] W. Dai, R. Nassar, A finite difference scheme for solving a threedimensional heat transport equation in a thin film with microscale thickness, INT J Numer Meth Eng 50 (2001) 1665.

[7] W. Dai, F. Han, Z. Sun, Accurate numerical method for solving dualphase-lagging equation with temperature jump boundary condition in nano heat conduction, Int. J. Heat Mass Transfer 64 (2013) 966. 
[8] J. Ghazanfarian, Z. Shomali, A. Abbassi, Macro-to Nanoscale Heat and Mass Transfer: The Lagging Behavior, Int J Thermophys 36 (2015) 1416.

[9] S. Both, B. Czl, T. Fülöp, G. Grf, A. Gyenis, R. Kovcs, P. Vn, J. Verhs, Deviation from the Fourier law in room-temperature heat pulse experiments, J. Non-Equilib. Thermodyn. 41.1. (2016) 41-48 .

[10] B. Li, J. Wang, Anomalous heat conduction and anomalous diffusion in one-dimensional systems, Phys. Rev. Lett. 91.4 (2003) 044301.

[11] C-W. Chang, D. Okawa, H. Garcia, A. Majumdar, A. Zettl, Breakdown of Fouriers law in nanotube thermal conductors, Phys. Rev. Lett. 101.7 (2008) 075903.

[12] K. Hippalgaonkar, B. Huang, R. Chen, K. Sawyer, P. Ercius, A. Majumdar, Fabrication of microdevices with integrated nanowires for investigating low-dimensional phonon transport, Nano Lett. 10.11 (2010) 4341-4348.

[13] M. Fujii, X. Zhang, H. Xie, H. Ago, K. Takahashi, T. Ikuta, H. Abe, T. Shimizu, Measuring the thermal conductivity of a single carbon nanotube, Phys. Rev. Lett. 95.6 (2005) 065502.

[14] N. Yang, G. Zhang, and B. Li, Violation of Fourier's law and anomalous heat diffusion in silicon nanowires, Nano Today 5.2 (2010) 85-90.

[15] G. Chen, Ballistic-diffusive heat-conduction equations, Phys. Rev. Lett. 86.11 (2001) 2297. 
[16] F.X. Alvarez, D. Jou, Memory and nonlocal effects in heat transport: from diffusive to ballistic regimes, Appl. Phys. Lett. 90.8 (2007) 083109.

[17] Y. Dong, B-Y Cao, Z-Y Guo, Generalized heat conduction laws based on thermomass theory and phonon hydrodynamics, J Appl Phys 110.6 (2011) 063504.

[18] H. Basirat, J. Ghazanfarian, P. Forooghi, Implementation of dual-phaselag model at different Knudsen numbers within slab heat transfer, in: Proceedings of International Conference on Modeling, Simulation (MS06), August, Konia, Turkey, 895, (2006).

[19] M. Moghaddam, J. Ghazanfarian, A. Abbassi, Implementation of DPLDD model for the simulation of nanoscale MOS devices, IEEE Trans. Electron Devices 61 (2014) 3131.

[20] R. Saghatchi, J. Ghazanfarian, A Novel SPH Method for the Solution of Dual-Phase-Lag Model with Temperature-Jump Boundary Condition in Nanoscale, Appl Math Model (2014) DOI: 10.1016/j.apm.2014.07.025.

[21] D.Y. Tzou, K.S. Chiu, Temperature-dependent thermal lagging in ultrafast laser heating, Int. J. Heat Mass Transfer 44 (2001) 1725.

[22] H. Wang, W. Dai, R. Melnik, A finite difference method for studying thermal deformation in a double-layered thin film exposed to ultrashort pulsed lasers, Int. J. Therm. Sci. 45 (2006) 1179.

[23] K. Ramadan, W.R. Tyfour, and M.A. Al-Nimr, On the Analysis of Short-Pulse Laser Heating of Metals Using the Dual Phase Lag Heat Conduction Model, Journal of heat transfer 131 (2009) 111301. 
[24] S. Kumar, S. Bag, M. Baruah, Finite element model for femtosecond laser pulse heating using dual phase lag effect, J Laser Appl 28.3 (2016) 032008.

[25] J. Zhou, Y. Zhang, J.K. Chen, An axisymmetric dual-phase-lag bioheat model for laser heating of living tissues, Int. J. Therm. Sci. 48 (2009) 1477.

[26] D.Y. Tzou, Lagging behaviour in biological systems, Journal of heat transfer 134 (2012) 051006.

[27] B. Kundu, Exact analysis for propagation of heat in a biological tissue subject to different surface conditions for therapeutic applications, Appl Math Comput. 285 (2016) 204-216.

[28] J. Shiomi, S. Maruyama, Non-Fourier heat conduction in a single-walled carbon nanotube: classical molecular dynamics simulations, Phys. Rev. B 73 (2006) 205420.

[29] J. Shiomi, S. Maruyama, Heat conduction of single-walled carbon nanotube isotope superlattice structures: A molecular dynamics study, Phys. Rev. B 74 (2006) 155401.

[30] A. Volkov, T. Shiga, D. Nicholson, J. Shiomi, L. Zhigilei, Effect of bending buckling of carbon nanotubes on thermal conductivity of carbon nanotube materials, J. Appl. Phys. 111 (2012) 053501.

[31] J. Zhang, X. Huang, Y. Yue, J. Wang, X. Wang, Dynamic response of graphene to thermal impulse, Phys. Rev. B 84 (2011) 235416. 
[32] R. Yang, G. Chen, M. Laroche, Y. Taur, Multidimensional transient heat conduction at nanoscale using the ballistic-diffusive equations and the Boltzmann equation, ASME J. Heat Transfer 127 (2005) 298.

[33] J. Ghazanfarian, A. Abbassi, Effect of boundary phonon scattering on Dual-Phase-Lag model to simulate micro- and nano-scale heat conduction, Int. J. Heat Mass Transfer 52 (2009) 3706.

[34] J. Ghazanfarian, Z. Shomali, Investigation of dual-phase-lag heat conduction model in a nanoscale metal-oxide-semiconductor field-effect transistor, Int. J. Heat Mass Transfer 55 (2012) 6231.

[35] F. Nasri, M.F. Ben Aissa, M.H. Gazzah, H. Belmabrouk, 3-D thermal conduction in a nanoscale Tri-Gate MOSFET based on Single-PhaseLag model, App Therm Eng. 91 (2015) 647.

[36] F. Nasri, M.F. Ben Aissa, H. Belmabrouk, Effect of second-order temperature jump in Metal-Oxide-Semiconductor Field Effect Transistor with Dual-Phase-Lag model, microelectr J 46 (2015) 67-74.

[37] G. Fiori, G. Iannaccone, Simulation of graphene nanoribbon field-effect transistors, IEEE Trans. Electron Devices 28 (2007) 760-762.

[38] J.G. Son, M. Son, K.J. Moon, B.H. Lee, J.M. Myoung, M.S. Strano, M.H. Ham, C.A. Ross, Sub10 nm Graphene Nanoribbon Array FieldEffect Transistors Fabricated by Block Copolymer Lithography, Adv. Mater. 25 (2013) 4723-4728. 
[39] S. Subrina, D. Kotchetkov, A.A. Balandin, Heat removal in silicon-oninsulator integrated circuits with graphene lateral heat spreaders, IEEE Trans. Electron Devices, 30.12 (2009) 1281-1283.

[40] S. Subrina, Heat transport in graphene interconnect networks with graphene lateral heat spreaders, Nanotechnology, IEEE T Nanotechnol 11.4 (2012): 777-781.

[41] A. Sellitto, F.X. Alvarez, Non-Fourier heat removal from hot nanosystems through graphene layer, Nanoscale Systems: Mathematical Modeling, Theory and Applications 1 (2012) 38-47.

[42] A. Suszko, M.S. El-Genk, Thermally anisotropic composite heat spreaders for enhanced thermal management of high-performance microprocessors, Int. J. Therm. Sci. 100 (2016) 213-228.

[43] R.S. Samian, A. Abbassi, J. Ghazanfarian, Thermal Investigation of Common 2D FETs and New Generation of 3-D FETs using Boltzmann Transport Equation in Nanoscale, Int. J. Mod Phys C 24 (2013) 1350064.

[44] C. Cattaneo, A form of heat conduction equation which eliminates the paradox of instantaneous propagation, Compte Rendus 247 (1958) 431.

[45] P. Vernotte, Les paradoxes de la throrie continue de lrquation de la chaleur, Compte Rendus 246 (1958) 3154.

[46] D.Y. Tzou, A unified field approach for heat conduction from macro-to microscales, ASME J. Heat Transfer 117 (1995) 8. 
[47] D.Y. Tzou, Macro-to-Microscale Heat Transfer- The Lagging behaviour, Taylor \& Francis, New York, 1997.

[48] Z. Shomali, A. Abbassi, Investigation of highly non-linear dual-phase-lag model in nanoscale solid argon with temperature-dependent properties, Int. J. Therm. Sci. 87 (2014) 56.

[49] Z. Shomali, J. Ghazanfarian, A. Abbassi, Effect of film properties for non-linear DPL model in a nanoscale MOSFET with high-k material: $\mathrm{ZrO}_{2} / \mathrm{HfO}_{2} / \mathrm{La}_{2} \mathrm{O}_{3}$, Superlattice Microst 83 (2015) 699.

[50] K.E. Goodson, M.I. Flik, Effect of microscale thermal conduction on the packing limit of silicon-on-insulator electronic devices, IEEE Trans. Compon. Hybrids Manuf. Technol. 15 (1992) 715-722. 


\section{List of Tables}

1 Mesh-time step independency check . . . . . . . . . . . 33

2 The thermal properties of silicon [32] and silicon-dioxide [50]

at $T=300 \mathrm{~K} \ldots \ldots \ldots \ldots$ 


\section{List of Figures}

1 Schematic of geometry (a) The common 3-D silicon MOSFET, (b) The Fin-field effect transistor, (c) The typical Tri-Gate

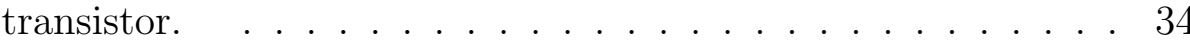

2 (a) The comparison of temperature profiles obtained from the present study with the result presented in Ghazanfarian and Shomali [34] at $t=10 \mathrm{ps,} \mathrm{(b)} \mathrm{The} \mathrm{heat} \mathrm{flux} \mathrm{versus} z$ over center-line for $t=10 \mathrm{ps} . \ldots \ldots \ldots \ldots$

3 (a) The temperature distribution of the FinFET with constant thermal properties over center-line when $t=5 \mathrm{ps}$, (b) The heat flux profile for the same FinFET at $t=5 \mathrm{ps},(\mathrm{c}, \mathrm{d})$ the same as (a) and (b) but for $t=10$ ps. . . . . . . . . . . . 36

4 (a) The center-line temperature profile of the Fin-field effect transistor at $t=15 \mathrm{ps}$, (b) The Heat flux distribution along $\mathrm{z}$ direction at the center-line of the FinFET when $t=15$ ps. . 37

5 The heat flux plot of the FinFET at $t=20$ ps. . . . . . . 38

6 (a) The temperature and (b) the heat flux distributions for the FinFET with constant (CST) and temperature-dependent (TD) thermal parameters on heat generating region, (c,d) The same as $(\mathrm{a}, \mathrm{b})$ but for the FinFET in which the heat generation zone is switched on for the first 10 ps at $t=15$ ps. . . . . . 39 
7 (a) The temperature profiles for a Tri-Gate MOSFET with constant thermal characteristic at the center-line when $t=$ 5 ps, (b) Heat flux versus $z$ for a Tri-Gate transistor at $t=$ $5 \mathrm{ps}$, (c) The temperature distribution at $10 \mathrm{ps}$, (d) The same as (b) but for $t=10 \mathrm{ps}$ when $B=0.25$. . . . . . . . . 40

8 (a) The temperature and (b) the heat flux distributions along the center-line at $t=15 \mathrm{ps} \ldots \ldots \ldots \ldots \ldots$. . . . . 41

9 The heat flux profiles at the center-line when $t=20 \mathrm{ps}$ and $B=1.9 \ldots \ldots \ldots \ldots \ldots \ldots \ldots \ldots \ldots \ldots$

10 (a) The temperature profiles of the Tri-Gate with constant (CST) and temperature-dependent (TD) thermal parameters along the center-line at $t=5 \mathrm{ps}$, (b) The heat flux distribution for the Tri-Gate with CST and TD thermal specifications when $t=5 \mathrm{ps},(\mathrm{c}, \mathrm{d})$ the same as (a) and (b) but for $t=15 \mathrm{ps}$. . . 43

11 (a) The temperature distributions of the Tri-Gate MOSFET with and without the presence of the graphene heat spreader along the center-line at different times, (b) The heat flux distribution for the same transistors mentioned in (a). . . . . . . 44 
Table 1: Mesh-time step independency check

\begin{tabular}{lllll}
\hline number of grids in: & $\mathrm{x}-$ & $\mathrm{y}-$ & z-direction & time step size $(\mathrm{ps})$ \\
\hline Case I & 101 & 201 & 101 & 0.01 \\
Cases II-IV: channel & 22 & 201 & 101 & 0.01 \\
Cases II-IV: substrate & 101 & 201 & 101 & 0.01
\end{tabular}

Table 2: The thermal properties of silicon [32] and silicon-dioxide [50] at $T=300 \mathrm{~K}$.

\begin{tabular}{lllll}
\hline & $|\mathbf{v}|\left(\mathrm{ms}^{-1}\right)$ & $k\left(\mathrm{Wm}^{-1} \mathrm{~K}^{-1}\right)$ & $C\left(\mathrm{Jm}^{-3} \mathrm{~K}^{-1}\right)$ & $\lambda(\mathrm{nm})$ \\
\hline $\mathrm{Si}$ & 3000 & 150 & $1.5 \times 10^{6}$ & 100 \\
\hline $\mathrm{SiO}_{2}$ & 5900 & 1.4 & $1.75 \times 10^{6}$ & 0.4 \\
\hline
\end{tabular}




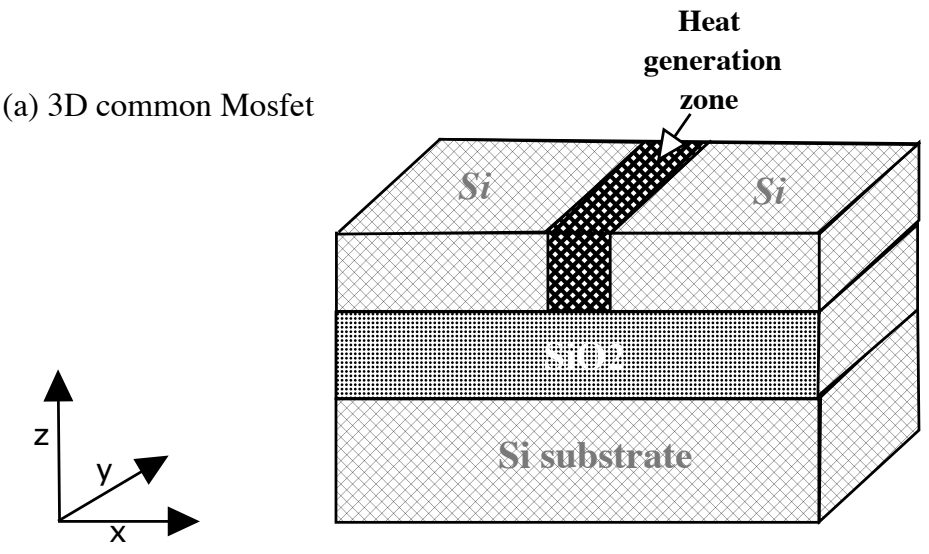

(b) FinFet Mosfet

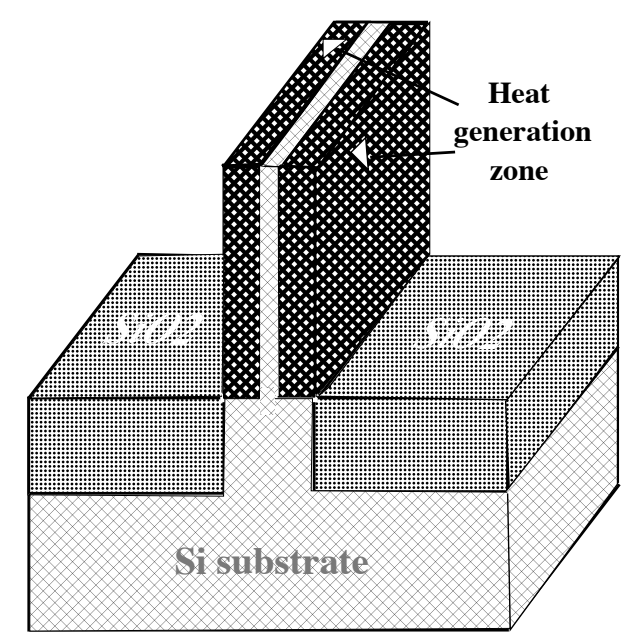

(c) Tri-gate Mosfet

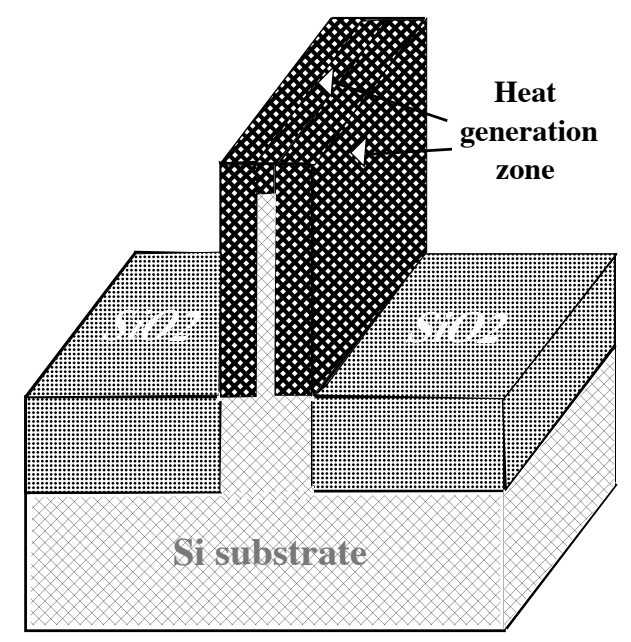

Figure 1: Schematic of geometry (a) The common 3-D silicon MOSFET, (b) The Fin-field effect transistor, (c) The typical Tri-Gate transistor. 

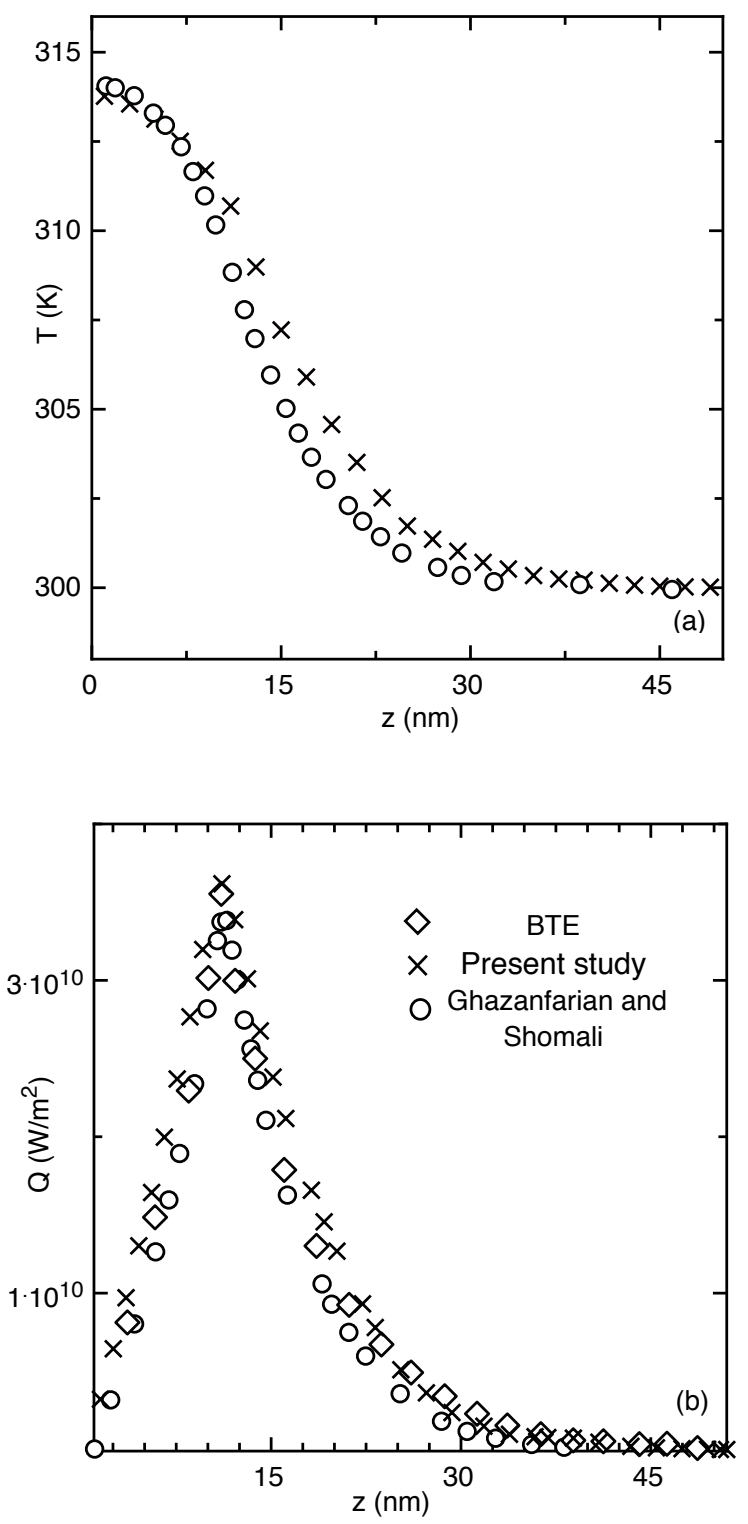

Figure 2: (a) The comparison of temperature profiles obtained from the present study with the result presented in Ghazanfarian and Shomali [34] at $t=10 \mathrm{ps}$, (b) The heat flux versus $z$ over center-line for $t=10 \mathrm{ps}$. 

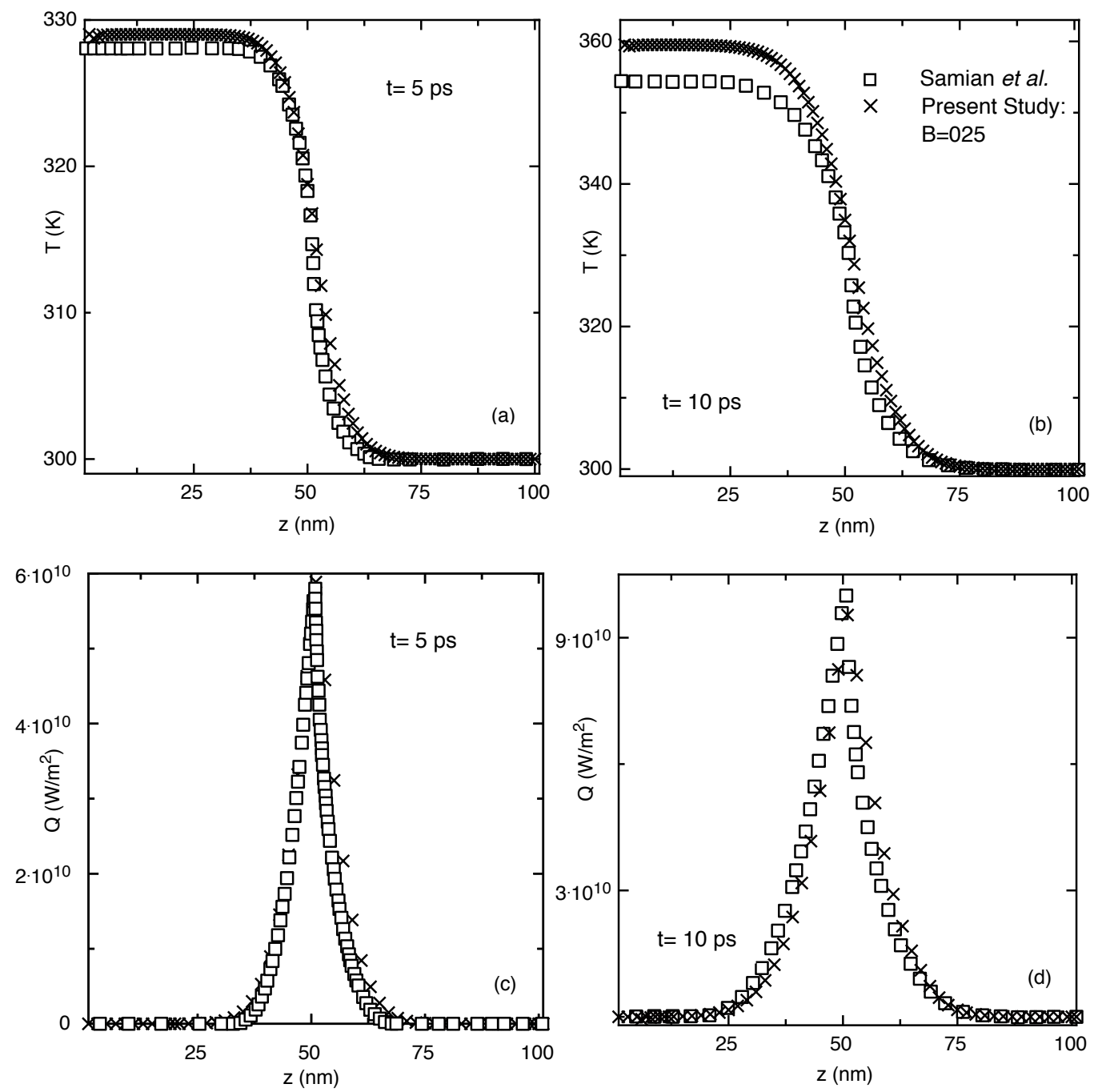

Figure 3: (a) The temperature distribution of the FinFET with constant thermal properties over center-line when $t=5 \mathrm{ps}$, (b) The heat flux profile for the same FinFET at $t=5 \mathrm{ps},(\mathrm{c}, \mathrm{d})$ the same as (a) and (b) but for $t=10 \mathrm{ps}$. 

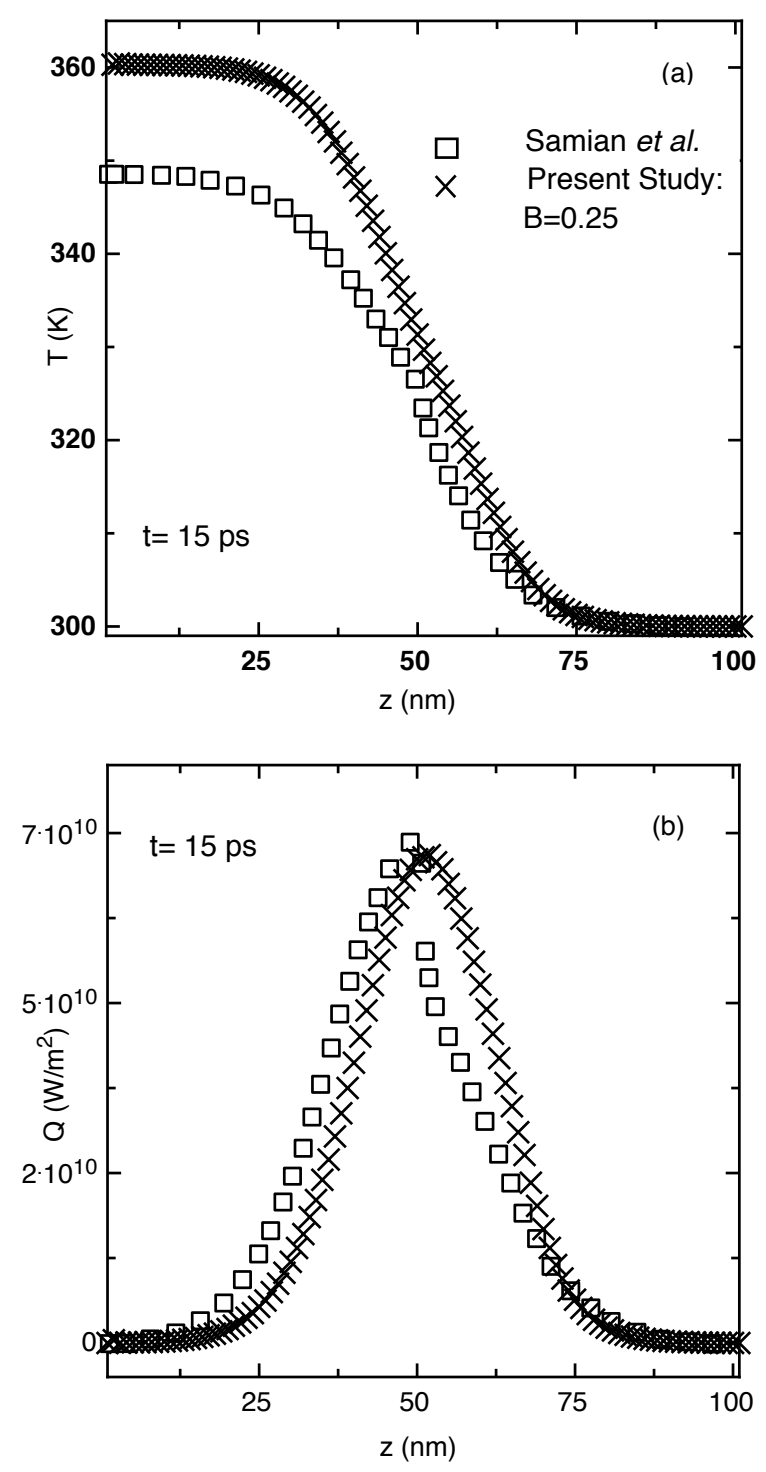

Figure 4: (a) The center-line temperature profile of the Fin-field effect transistor at $t=$ 15 ps, (b) The Heat flux distribution along $\mathrm{z}$ direction at the center-line of the FinFET when $t=15$ ps. 


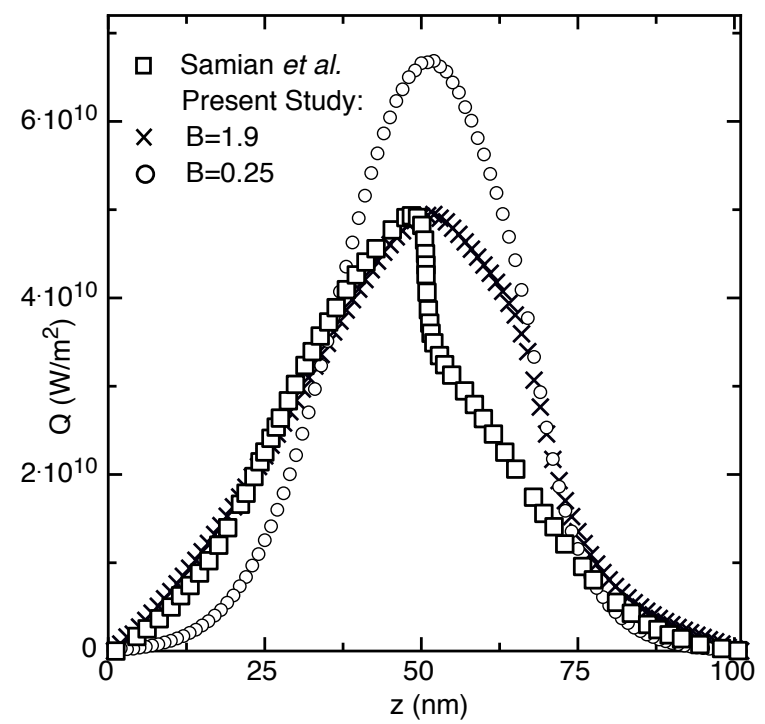

Figure 5: The heat flux plot of the FinFET at $t=20 \mathrm{ps}$. 

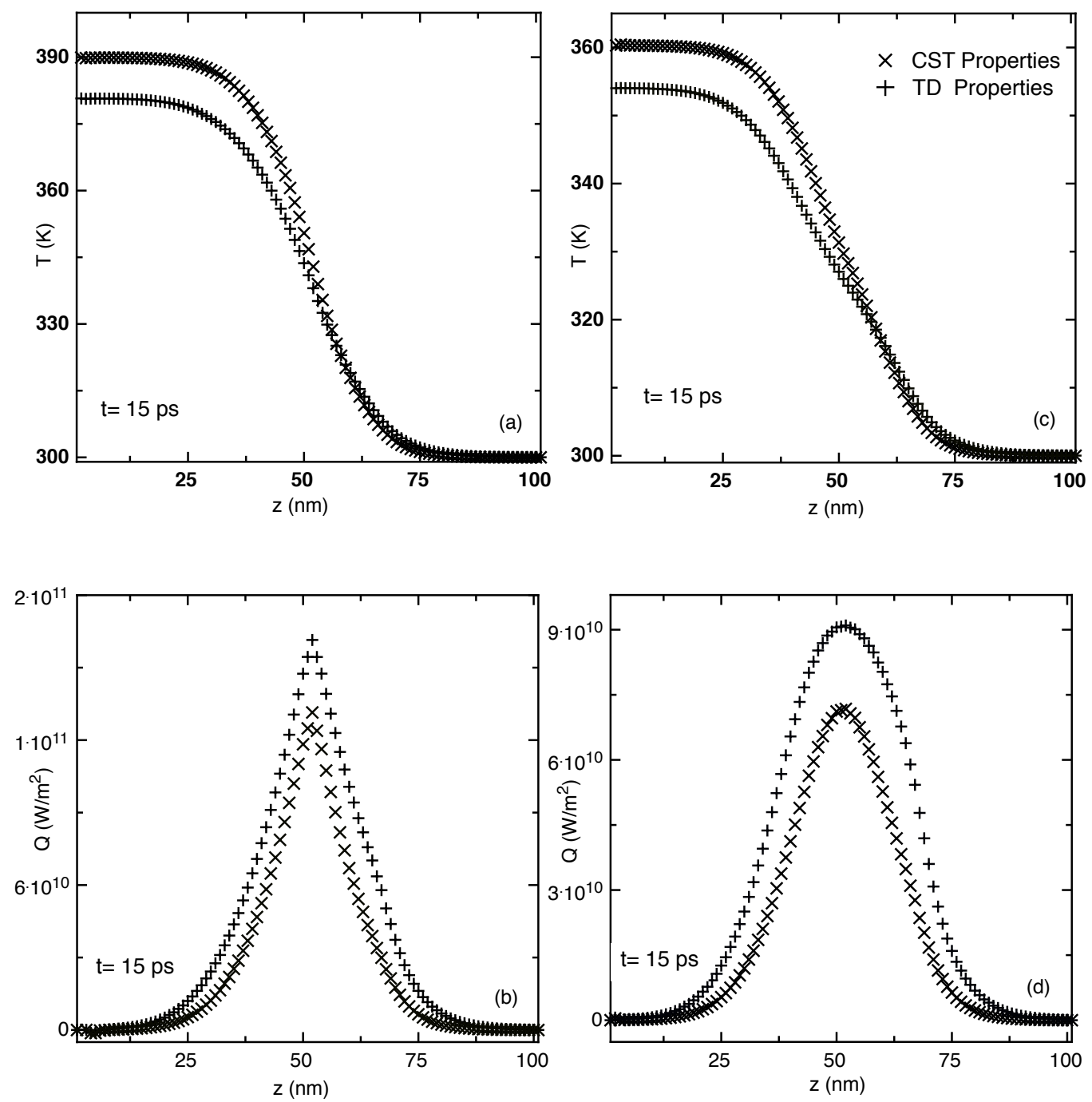

Figure 6: (a) The temperature and (b) the heat flux distributions for the FinFET with constant (CST) and temperature-dependent (TD) thermal parameters on heat generating region, $(\mathrm{c}, \mathrm{d})$ The same as $(\mathrm{a}, \mathrm{b})$ but for the FinFET in which the heat generation zone is switched on for the first $10 \mathrm{ps}$ at $t=15 \mathrm{ps}$. 

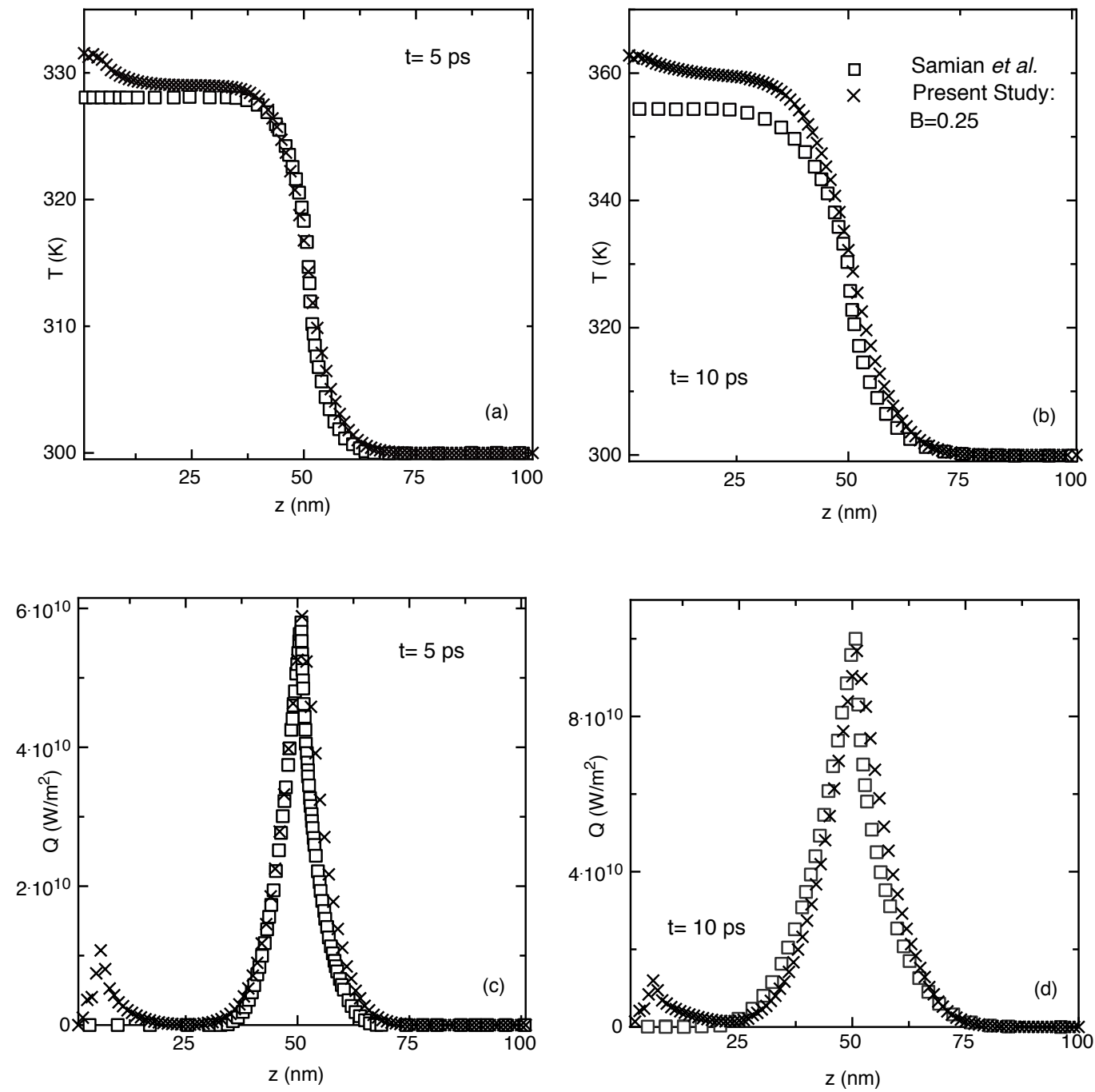

Figure 7: (a) The temperature profiles for a Tri-Gate MOSFET with constant thermal characteristic at the center-line when $t=5 \mathrm{ps}$, (b) Heat flux versus $z$ for a Tri-Gate transistor at $t=5 \mathrm{ps}$, (c) The temperature distribution at $10 \mathrm{ps}$, (d) The same as (b) but for $t=10 \mathrm{ps}$ when $B=0.25$. 

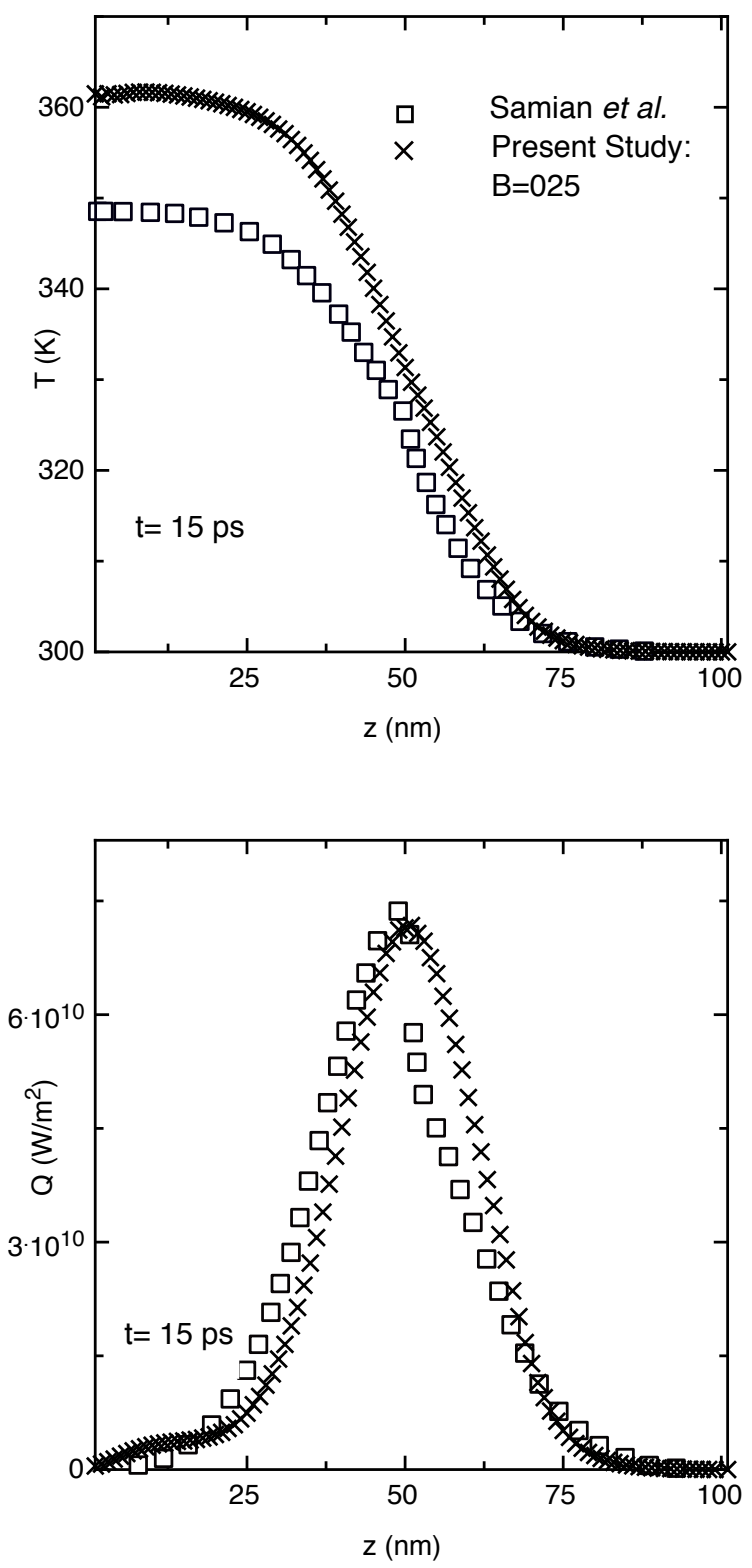

Figure 8: (a) The temperature and (b) the heat flux distributions along the center-line at $t=15 \mathrm{ps}$. 


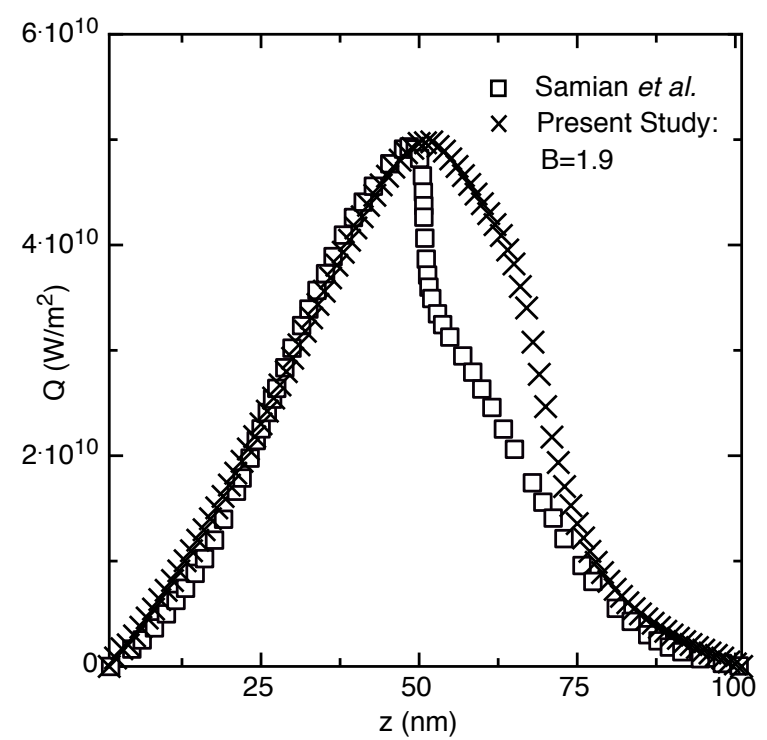

Figure 9: The heat flux profiles at the center-line when $t=20 \mathrm{ps}$ and $B=1.9$. 

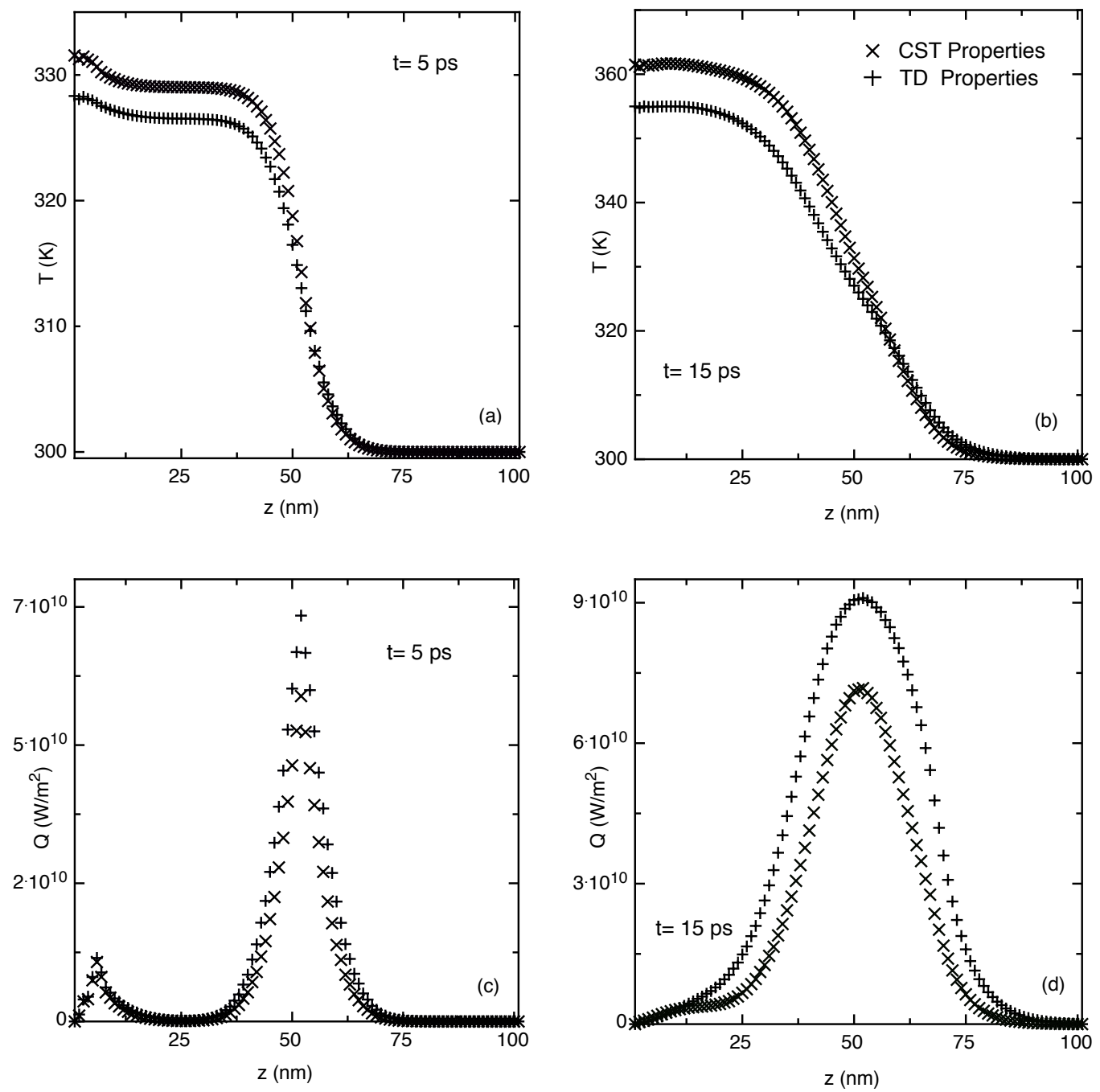

Figure 10: (a) The temperature profiles of the Tri-Gate with constant (CST) and temperature-dependent (TD) thermal parameters along the center-line at $t=5 \mathrm{ps}$, (b) The heat flux distribution for the Tri-Gate with CST and TD thermal specifications when $t=5 \mathrm{ps},(\mathrm{c}, \mathrm{d})$ the same as (a) and (b) but for $t=15 \mathrm{ps}$. 

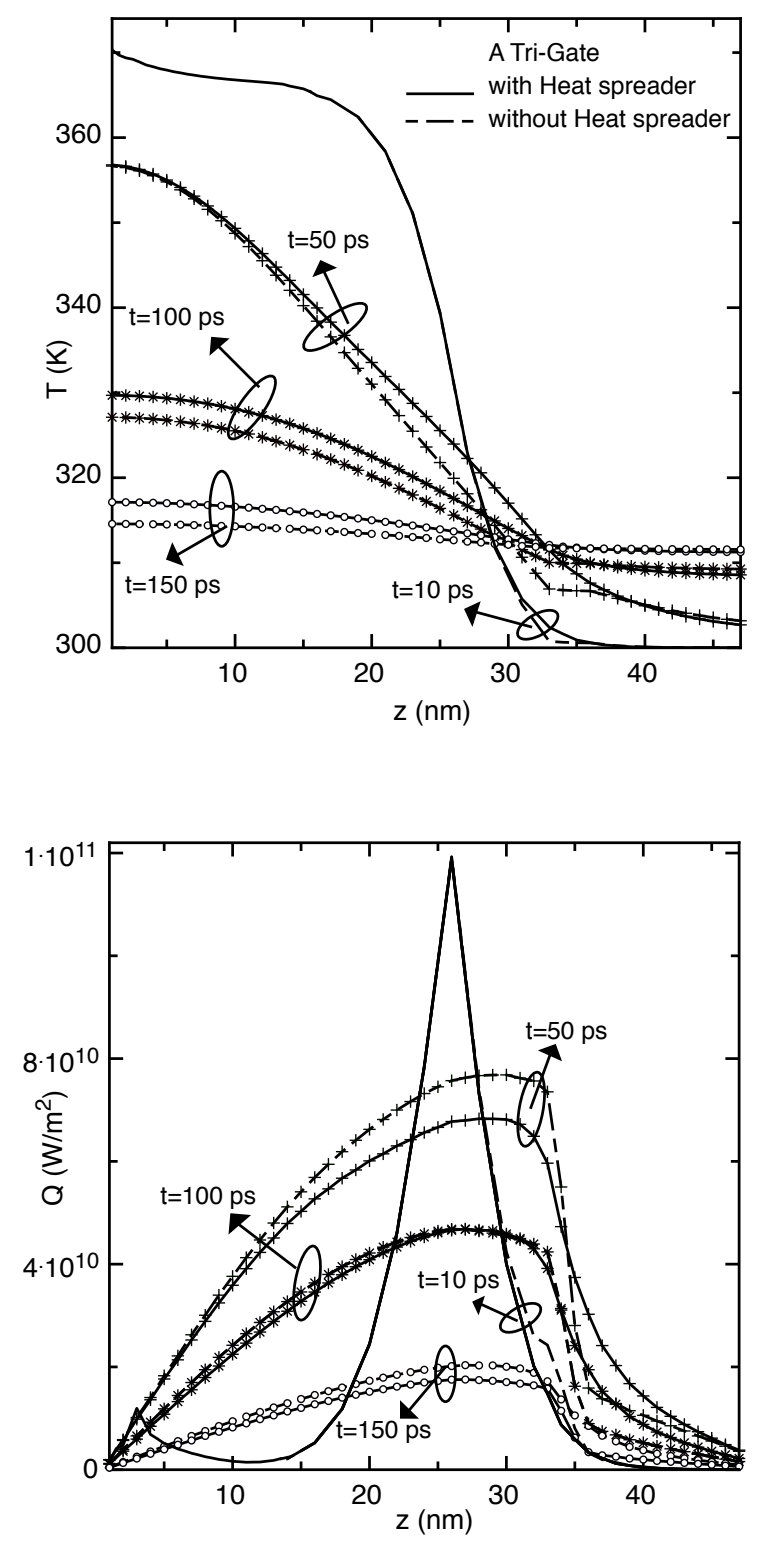

Figure 11: (a) The temperature distributions of the Tri-Gate MOSFET with and without the presence of the graphene heat spreader along the center-line at different times, (b) The heat flux distribution for the same transistors mentioned in (a). 